Research Paper

\title{
Assessment of the Long-term Diagnostic Performance of a New Serological Screening Scheme in Large-scale Nasopharyngeal Carcinoma Screening
}

\author{
Xia $\mathrm{Yu}^{1}$, Mingfang Ji1 ${ }^{1 凶}$, Weimin Cheng1, Biaohua $\mathrm{Wu}^{1}$, Yun $\mathrm{Du}^{1}{ }^{1,2}$, Sumei $\mathrm{Cao}^{2}$ \\ 1. Cancer Research Institute of Zhongshan City, Zhongshan, Guangdong Province, China \\ 2. Department of Cancer Prevention Research, Cancer Prevention Center, Sun Yat-sen University Cancer Center, State Key Laboratory of Oncology in \\ Southern China, Collaborative innovation Center for Cancer Medicine, Guangzhou, Guangdong Province, China \\ $\triangle$ Corresponding author: Mingfang Ji (jmftbh@sina.com) \\ (c) Ivyspring International Publisher. This is an open access article distributed under the terms of the Creative Commons Attribution (CC BY-NC) license \\ (https://creativecommons.org/licenses/by-nc/4.0/). See http://ivyspring.com/terms for full terms and conditions.
}

Received: 2017.11.09; Accepted: 2018.03.21; Published: 2018.05.24

\begin{abstract}
Background: Nasopharyngeal carcinoma (NPC) remains as a major public health burden in Southern China. Over the last decade, Epstein-Barr virus (EBV) serological detection has been the most promising tool used for NPC screening. The present study aims to evaluate the long-term diagnostic performance of a new NPC screening scheme (probability of NPC units [logit P], $P R O B \geq 0.65$ ), and compare this with other EBV seromarkers used within 2009-2015.

Methods: Enzyme-linked immunosorbent assay (ELISA) for EBV capsid antigen (VCA/lgA) and nuclear antigen-1 (EBNAl/lgA) was performed in 16,712 subjects, who were within 30-59 years old.All subjects were followed up for six years. The area under the receiver operating characteristic curve (AUC) and correlation analyses were preformed to evaluate the diagnostic value of different measures. Furthermore, the rates of early diagnosis in NPC patients were statistically analyzed.

Results: The new NPC screening scheme $(P R O B \geq 0.65)$ and the four strategies (VCA/lgA, EBNAl/lgA, VCA/lgA and EBNA1/lgA, and VCA/lgA or EBNA1/lgA) had comparable rates of early diagnosis for NPC (no significant difference was found), but the sensitivity of the new scheme (95.7\%) was higher than that of the others. The top three seromarkers with the largest AUC were $P R O B \geq 0.65$ (AUC:0.926, 95\% Cl: $0.885-0.966$ ), $V C A / \lg A$ or EBNAl/lgA (AUC:0.883, 95\% $\mathrm{Cl}: 0.824-0.942$ ), and EBNAI/lgA (AUC: $0.866,95 \% \mathrm{Cl}$ : 0.794-0.938).

Conclusion: The new NPC screening scheme (PROB $\geq 0.65)$ based on VCA/lgA and EBNA1/lgA outperforms the other seromarkers, and making it the preferred serodiagnostic strategy for long-term NPC screening in high-incidence areas.
\end{abstract}

Key words: Nasopharyngeal carcinoma, screening, Epstein-Barr antibodies, diagnosis

\section{Introduction}

Nasopharyngeal carcinoma (NPC) is prevalent in Southern China, and mainly affects the middle-aged population. NPC tends to present at an advanced stage at diagnosis, and has a relatively poor survival rate after diagnosis, because the primary anatomic site of the tumor is located in a silent area, and its symptoms are inconspicuous [1]. Despite the discovery of the very close association of EBV with $\mathrm{NPC}$, the exact role of this virus in NPC development has remained not completely elucidated. EBV serological detection has been the most promising tool used in NPC screening. However, most studies on EBV antibodies have been conducted on the basis of the small pilot cross-sectional study of preliminary NPC screening due to the lake of data obtained from large-scale samples from NPC long-term follow-ups. In the present study, a total of 16,712 participants were enrolled between 2009 and 2010. Enzyme-linked 
immunosorbent assay (ELISA) for $\mathrm{VCA} / \mathrm{IgA}$ and EBNA1/IgA were performed for all subjects, and the preliminary results of this serological screening were reported [2,3]. A six-year follow-up survey was carried out up to the end of December 2015. The purpose of the present study was to evaluate the long-term diagnostic performance of the new NPC screening scheme, and compare this with other seromarkers.

\section{Materials and Methods}

\section{Study population}

A serological follow-up study of NPC in Zhongshan City, Southern China, which is a high-incidence area of NPC, was conducted. A total of 16,712 individuals, who were 30-59 years old, were recruited between August 2009 and July 2010. A follow-up survey was carried out up to the end of December 2015.

\section{Serologic test and screening protocol}

Serum samples collected during enrollment and on subsequent occasions, and were tested in separate batches. The samples were stored at $4^{\circ} \mathrm{C}$ for use within one month, or stored at $-80^{\circ} \mathrm{C}$ for longer periods. Two screening markers, VCA/IgA (Euroimmun, Lubeck, Germany) and EBNA1/IgA (Zhongshan Bio-tech, Zhongshan, China), were tested by ELISA. The levels of these seromarkers were assessed by photometric measurement, according to manufacturers'instructions, and standardized by calculating the ratio of the optical density (OD) of the sample over that of the reference control (rOD). If the specific rOD was greater than 1 , the sample was regarded as positive [4].

The new combination of VCA/IgA and EBNA1/IgA was identified, which had a sensitivity of $92.8 \%$, a specificity of $91.6 \%$, and a ROC of 0.97 . Then, the prediction formula was developed, as follows: $\operatorname{Logit} P R O B=-3.934+2.203 \times \mathrm{VCA} / \mathrm{IgA}+4.797 \times$ EBNA1/IgA [5]. On the basis of a predefined serologic algorithm, the participants were classified into three subgroups: high-risk (PROB $\geq 0.98$ ), medium-risk $(0.98>\mathrm{PROB} \geq 0.65)$, and low-risk (PROB $<0.65)$. Except for patients diagnosed with NPC, the remaining high-risk and medium-risk subjects were followed-up annually, and blood samples were obtained to examine for EBV-related antibodies. Fiberoptic endoscopy was performed by local otorhinolaryngologists in Zhongshan People's Hospital when the serologic result reached the definition of high-risk. Nasopharyngeal biopsies were also performed when suspicious lesions were observed during the endoscopy procedure. Subjects in the low-risk group were reexamined every four years after the initial round of screening. NPC cases were identified by the research team among the high-risk group. As a complement, these cases were identified through linkage to the cancer registries, annually. NPC diagnoses from the low-risk group were mainly ascertained through the cancer registries. NPC was classified according to the World Health Organization classification [6], and tumor stage was defined according to the 2008 staging system of China [7]. Stage I and II were considered early stages.

\section{Statistical analysis}

During the screening, subjects with titer EBV antibodies were regarded as observed data, which include true positive a1, false negative b1, false positive $\mathrm{c} 1$ and true negative $\mathrm{d} 1$. That is, a1 represents NPC patients who are seropositive or high/medium risk subjects, b1 represents NPC patients who are seronegtive or low risk subjects, $\mathrm{c} 1$ represents healthy people who are seropositive or high/medium risk subjects, and $\mathrm{d} 1$ represents healthy people who are seronegtive or low risk subjects. Based on this notation, the formulas used to calculate for the sensitivity and specificity were as follows: Sensitivity $=\mathrm{a} 1 / \mathrm{a} 1+\mathrm{b} 1 ;$ Specificity $=\mathrm{d} 1 / \mathrm{c} 1+\mathrm{d} 1 ;$ Positive predictive value $=a 1 / a 1+c 1$.

The rates for the early diagnosis of NPC were compared using chi-square tests. The efficacy of EBV antibodies was evaluated using the sensitivity, specificity, positive predictive value (PPV), rate of missed diagnosis, Youden index and AUC. All statistical analyses were two-sided. A $P$-value $<0.05$ was considered statistically significant. All analyses were performed using SPSS 20.0 software.

\section{Results}

\section{Characteristics of screening participants}

Within the first turn of serological screening in 2009 and 2010, 401 and 1,024 out of 16,712 subjects were classified as high-risk and medium-risk, respectively. Thus a total of 1,425 subjects needed to be reexamined for the following two years. In fact, 972 and 623 of these subjects were reexamined in 2011 and 2012, respectively, and compliance decreased from $68.2 \%$ to $43.7 \%$. In 2013, 229 and 702 out of 10,801 subjects were classified as high-risk and medium-risk, respectively. Thus, a total of 931 subjects needed to be reexamined for the following two years. In fact, 749 and 502 of these patients were reexamined in 2013 and 2014, respectively, and compliance decreased from $80.5 \%$ to $53.9 \%$. During the two screening cycles of the NPC screening (six years), a total of 47 NPC patients were diagnosed from the study subjects. Among these 47 patients, 44 patients, who were classified as 
high-risk and medium-risk populations, were identified during the routine screening, while three patients, who were classified as a low-risk population, were identified through linkage to cancer registries. As shown in Table 1, the annual NPC incidences in the 30-59 year-old screening population were very high during the first turn of the serological screening and the first year of follow-up after the initial screening.

Table 1. Number of subjects tested and NPC detected during the screening

\begin{tabular}{|c|c|c|c|c|c|}
\hline Years & $\begin{array}{l}\text { Total } \\
\text { number }\end{array}$ & $\begin{array}{l}\text { No. of } \\
\text { high-risk and } \\
\text { medium-risk } \\
\text { patients }\end{array}$ & Compliance & $\begin{array}{l}\text { No. of } \\
\text { patients } \\
\text { with } \\
\text { NPC }\end{array}$ & $\begin{array}{l}\text { Annual NPC } \\
\text { incidence per } \\
100,000 \\
\text { person-year }\end{array}$ \\
\hline $\begin{array}{l}\text { Preliminary } \\
\text { screening between } \\
2009 \text { and } 2010\end{array}$ & 16,712 & 1,425 & - & 25 & 149.59 \\
\hline Follow-up in 2011 & & 972 & $68.2 \%$ & 14 & 65.82 \\
\hline Follow-up in 2012 & & 623 & $43.7 \%$ & 2 & 11.97 \\
\hline Follow-up in 2013 & 10,801 & 931 & - & 1 & 5.98 \\
\hline Follow-up in 2014 & & 749 & $80.5 \%$ & 3 & 11.97 \\
\hline Follow-up in 2015 & & 502 & $53.9 \%$ & 2 & 5.98 \\
\hline
\end{tabular}

Note: One patient, who was classified as a low-risk population, was identified with NPC through linkage to the cancer registries in 2011, 2013 and 2015, respectively.

\section{Diagnostic values of the new NPC screening scheme (PROB $\geq 0.65)$ and the other four seromarkers in the preliminary and follow-up study}

The sensitivity, specificity and AUC of the individual seromarkers varied between $\mathrm{VCA} / \mathrm{IgA}$ and EBNA1/IgA, with EBNA1/IgA outperforming VCA/IgA. The sensitivity, specificity, PPV, rate of missed diagnosis, Youden index and AUC was $57.4 \%$, $94.3 \%, 2.8 \%, 42.6 \%, 51.7 \%$ and 0.759 , respectively, for $\mathrm{VCA} / \operatorname{IgA}$, and $76.6 \%, 96.2 \%, 5.3 \%, 23.4 \%, 72.8 \%$ and 0.866 , respectively, for EBNA1/IgA.

For the combinations of seromarkers $\mathrm{VCA} / \mathrm{IgA}$ and EBNA1/IgA, the seropositivity for both VCA/IgA and EBNA1/IgA was considered as a positive screening test result, while the seronegtivity for either VCA/IgA or EBNA1/IgA was considered as a negative result. For VCA/IgA or EBNA1/IgA, the seropositivity for either $\mathrm{VCA} / \operatorname{IgA}$ or EBNA1/IgA was considered as a positive result, while the seronegtivity for both $\mathrm{VCA} / \operatorname{Ig} \mathrm{A}$ and EBNA1/IgA was considered as a negative result. $P R O B \geq 0.65$ had the highest classification accuracy, with a sensitivity of $95.7 \%$, a specificity of $91.6 \%$, a PPV of $3.1 \%$, a rate of missed diagnosis of $4.3 \%$ and a Youden index of $87.3 \%$. The top three seromarkers with the largest AUC were $P R O B \geq 0.65$ (AUC: 0.926, 95\% CI: 0.885-0.966), VCA/IgA or EBNA1/IgA (AUC: 0.883, 95\% CI: 0.824-0.942), and EBNA1/IgA (AUC: 0.866, 95\% CI: 0.794-0.938) (Table 2 and Figure 1).

\section{Analysis of the rate of early diagnosis for the new NPC screening scheme and the other four seromarkers in NPC screening}

The rates of early diagnosis for $\mathrm{VCA} / \mathrm{IgA}$, EBNA1/IgA, VCA/IgA and EBNA1/IgA, VCA/IgA or EBNA1/IgA, and $\mathrm{PROB} \geq 0.65$ were $82.1 \%(23 / 28)$, $74.3 \%$ (26/35), $78.3 \%$ (18/23), $77.5 \%(31 / 40)$ and $79.5 \%$ (35/44), respectively. No significant difference was found in the rate of early diagnosis among the five groups $(P>0.05)$. However, as shown above, $P R O B \geq 0.65$ can identify the most NPCs (44) and the most early-stage NPCs (35).

\section{Discussion}

Zhongshan City in Southern China is one of high-incidence areas of NPC [8]. Since the 1980s, the Research Institute of Zhongshan City has launched several pilot efforts to conduct NPC mass screening by measuring two traditional seromarkers, VCA/IgA and IgA antibodies against EBV early antigen (EA/IgA), by using immunofluorescence assay. The limitations of relatively low PPV, lack of a standardized method, high intraobserver variations, and time-consuming protocols make it less applicable in large-scale population screenings [9]. A previous study [5] used a logistic regression model to identify an optimal biomarker panel to discriminate NPC from controls. It was revealed that the new combination test for $\mathrm{VCA} / \operatorname{Ig} \mathrm{A}$ and EBNA1/IgA by ELISA outperformed the traditional NPC screening scheme, in terms of both sensitivity and specificity. The Research Institute of Zhongshan City launched the new serological screening for 16,712 subjects in 2009 and 2010, and a follow-up survey was carried out up to the end of December 2015. The purpose of the present study was to evaluate the long-term diagnostic performance of the new NPC screening scheme, and compare it with other seromarkers.

Table 2. Diagnostic accuracy of individual and combinations of seromarkers

\begin{tabular}{|c|c|c|c|c|c|}
\hline Group & Sensitivity & Specificity & Positive predictive value & Rate of missed diagnosis & Youden index \\
\hline VCA/IgA & $57.4 \%$ & $94.3 \%$ & $2.8 \%$ & $42.6 \%$ & $51.7 \%$ \\
\hline EBNA1/IgA & $76.6 \%$ & $96.2 \%$ & $5.3 \%$ & $23.4 \%$ & $72.8 \%$ \\
\hline VCA/IgA and EBNA1/IgA & $48.9 \%$ & $99.5 \%$ & $2.3 \%$ & $51.5 \%$ & $48.4 \%$ \\
\hline VCA/IgA or EBNA1/IgA & $85.1 \%$ & $90.1 \%$ & $2.6 \%$ & $14.9 \%$ & $75.2 \%$ \\
\hline$P R O B \geq 0.65$ & $95.7 \%$ & $91.6 \%$ & $3.1 \%$ & $4.3 \%$ & $87.3 \%$ \\
\hline
\end{tabular}


ROC Curve

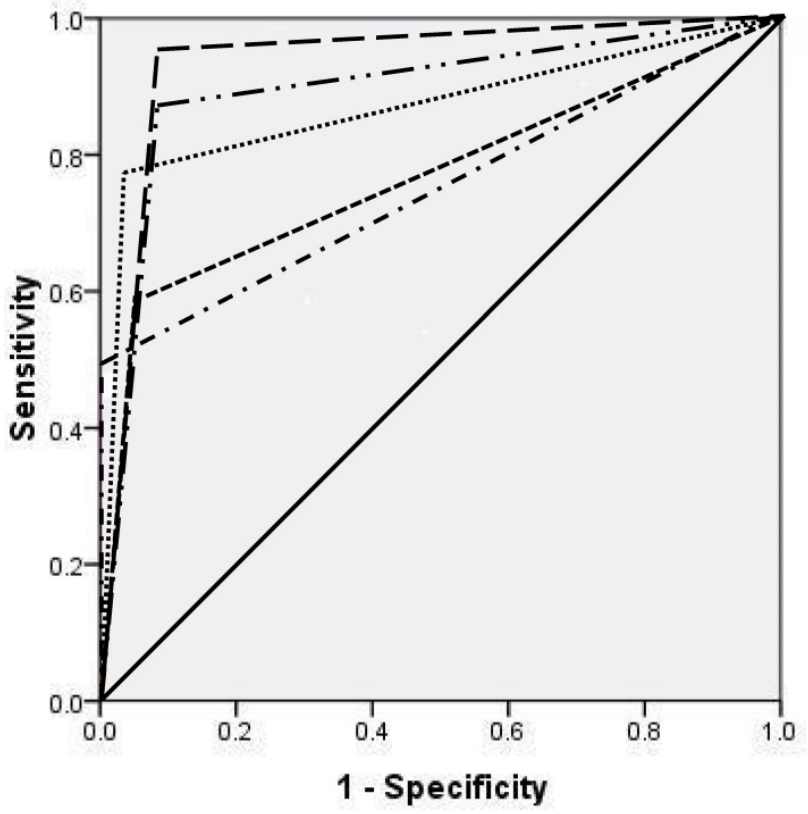

Source of the Curve

----- $V C A$

...........EBNA1

- $-\mathrm{PROB}$

- - VCAandEBNA1

- ..v VAorEBNA1

Reference Line

Figure 1. ROCs for VCA/lgA, EBNA1/lgA and their combinations. The AUC of VCA/lgA was 0.759 ( $95 \% \mathrm{Cl}: 0.672-0.846$ ), the AUC of EBNA1/lgA was 0.866 ( $95 \%$ $\mathrm{Cl}$ : $0.794-0.938$ ), the AUC of VCA/lgA and EBNAl/lgA was 0.742 (95\% Cl: $0.650-0.835)$, the AUC of VCA/lgA or EBNAl/lgA was 0.883 (95\% Cl: $0.824-0.942)$, and the $A \cup C$ of $P R O B \geq 0.65$ was 0.926 ( $95 \% \mathrm{Cl}: 0.885-0.966)$.

In the serological screening of the present study, 44 NPC patients were detected in high-risk and medium-risk populations in the two screening cycles. Among these patients, 35 patients were in stage I or II. Furthermore, the rate of early diagnosis was $79.5 \%$, which was significantly higher than those in clinic [10]. In the preliminary screening and during the first year of follow-up, the average annual incidence in the cohort was $149.59 / 10^{5}$ and $83.90 / 10^{5}$, respectively, when compared with the 30-59 year-old general population $\left(29.03 / 10^{5}\right)$. This was based on the registered data of the Cancer Institute of Zhongshan City. The screening programme resulted in more diagnosis and early NPCs, when compared to the general population. The overall survival for NPC has increased in recent years $[11,12]$. Hence, prospective randomized trials are needed to provide sound evidence for the potential effect of screening in reducing NPC-specific mortality [13].

On the basis of the 6-year follow-up period for the NPC screening, the diagnostic value of the new NPC screening scheme $(P R O B \geq 0.65)$ and the other four seromarkers were investigated by ELISA for NPC detection, including VCA/IgA, EBNA1/IgA, VCA/IgA and EBNA1/IgA, and VCA/IgA or EBNA1/IgA. The results revealed that there was no significant difference in the rate of early diagnosis for EBV seromarkers. For individual seromarkers, the diagnostic performance of EBNA1/IgA was superior to VCA/IgA, with an AUC of 0.866 , a PPV of $5.3 \%$, and a specificity of $96.2 \%$. However, its sensitivity was only $76.6 \%$, and the rate of missed diagnosis was unsatisfactory. For the combinations of seromarkers, the diagnostic performance of $\mathrm{VCA} / \mathrm{IgA}$ or EBNA1/IgA was superior to $\mathrm{VCA} / \operatorname{IgA}$ and EBNA1/IgA, with a sensitivity of $85.1 \%$ and a PPV of $2.6 \%$, leading to unnecessary fiberoptic endoscopy, as well as psychological pressures associated with the procedure. The new NPC screening scheme $(\mathrm{PROB} \geq 0.65)$ resulted in a decrease in missed diagnoses for subjects in the NPC screening, with a sensitivity of $95.7 \%$, a specificity of $91.6 \%$, a PPV of $3.1 \%$, and an AUC of 0.926. This increased the accuracy of the large-scale NPC screening, and can be the preferred serodiagnositc strategy for first turn serological screening and the yearly follow-up.

One of the principles for cancer screening is the follow-up. The screening interval for NPC is a controversial issue that has been inadequately evaluated. Rao DP et al. [14] constructed a Markov simulation model based on the natural history of NPC to evaluate different screening interval strategies for NPC screening. In the present study, it was found that 93.6\% (44/47) NPCs were detected from high-risk and medium-risk populations. Furthermore, the new serological screening was preferable for NPC screening. However, 38 of $44(86.4 \%)$ NPCs were detected during the preliminary serological screening and the first year of follow-up. The NPC detection rate has been dropping significantly ever since. 
The present study has several limitations. First, the screening compliance was unsatisfactory. Second, not all high-risk participants underwent nasopharyngeal endosocopy or biopsy, which may cause bias and impact the final results.

In conclusion, the use of a combined test of EBV antibodies by ELISA is recommended to identify NPC patients. The logistic regression model that combined VCA/IgA with EBNA1/IgA was selected to identify the probability of suffering from NPC. It is reasonable to consider that the new serological screening could increase NPC diagnostic accuracy. However, the diagnostic performance has decreased since the first year of follow-up. Future work is warranted to develop a more reasonable screening interval, in order to enhance NPC screening efficiency in the follow-up protocol [15].

\section{Acknowledgements}

This research was supported by grants from the National Science Foundation of China (No. 81572062), the 20 ${ }^{\text {th }}$ National Science and Technology Support Program of China (2014BAI09B10), the Sun Yat-sen University Clinical Research 5010 Program (2013012), and the Early Detection of Cancer Project in China (2010-13).

\section{Competing Interests}

The authors have declared that no competing interest exists.

\section{References}

1. Lee AW, Foo W, Law SC, et al. Nasopharyngeal carcinoma:presenting symptoms and duration before diagnosis. Hong Kong Med J. 1997;3(4):355-361.

2. Xia Yu, Mingfang JI, Yuanlong Yu, et al. Epidemiological Study of Epstein-barr Virus Infection for Nasopharyngeal Carcinoma Screening in High-incidence Areas [article in Chinese]. Chinese Journal of Clinical Oncology. 2011;38(24):1551-1554.

3. Liu Z, Ji MF, Huang QH, et al. Two Epstein-Barr virus-related serologic antibody tests in nasopharyngeal carcinoma screening: results from the initial phase of a cluster randomized controlled trial in Southern China. Am J Epidemiol. 2013;177(3):242-250.

4. Ming-Fang Ji,Qi-Hong Huang, Xia Yu,et al. Evaluation of plasma Epstein-Barr virus (EBV) DNA load to distinguish nasopharyngeal carcinoma patients from healthy high-risk population in Southern China. Cancer. 2014;120(9):1353-1360.

5. Liu Y,Huang Q, Liu W, et al. Establishment of VCA and EBNA1 IgA-based combination by enzyme-linked immunosorbent assay as preferred screening method for nasopharyngeal carcinoma: a two-stage design with a preliminary performance study and a mass screening in southern China. Int J Cancer. 2012;131(2):406-416.

6. Shanmugaratnam K, Sobin LH. The World Health Organization histological classification of tumours of the upper respiratory tract and ear. A commentary on the second edition. Cancer. 1993;71(8):2689-2697.

7. Lin ZX, Yang ZN, Zhan YZ, et al. Application study of the 2008 staging system of nasopharyngeal carcinoma [article in Chinese]. Ai Zheng. 2009;28(10):1029-1032.

8. Wei $\mathrm{K}, \mathrm{Xu} \mathrm{Y}$, Liu J, et al. No incidence trends and no change in pathological proportions of nasopharyngeal carcinoma in Zhongshan in 1970-2007. Asian Pac J Cancer Prev. 2010;11(6):1595-1599.

9. Karray H, Ayadi W, Fki L, et al. Comparison of three different serological techniques for primary diagnosis and monitoring of nasopharyngeal carcinoma in two age groups from Tunisia. J Med Virol. 2005;75(4):593-602.

10. Chan KC, Hung EC, Woo JK, et al. Early detection of nasopharyngeal carcinoma by plasma Epstein-Barr virus DNA analysis in a surveillance program. Cancer. 2013;119(10):1838-1844.
11. Qing Liu, Jin-Ou Chen, Qi-Hong Huang, et al. Trends in the survival of patients with nasopharyngeal carcinoma between 1976 and 2005 in Sihui, China: a population-based study. Chin J Cancer. 2013;32(6):325-333.

12. Huang TR, Zhang SW, Chen WQ, et al. Trends in nasopharyngeal carcinoma mortality in China, 1973-2005. Asian Pac J Cancer Prev. 2012;13(6):2495-2502.

13. Pepe MS, Etzioni R, Feng Z, et al. Phases of biomarker development for early detection of cancer. J Natl Cancer Inst. 2001;93(14):1054-1061.

14. Rao DP, Gu J, Meng $\mathrm{XH}$, et al. An efficacy analysis for nasopharyngeal carcinoma screening of different screening intervals. J Int Med Res. 2012;40(2): 525-536.

15. Lian $\mathrm{SF}, \mathrm{Ji} \mathrm{MF}, \mathrm{Wu} \mathrm{BH}$, et al. The following-up study of high-risk and moderate-risk groups defined by EB virus serology test at the nasopharyngeal carcinoma screening programme [article in Chinese]. Chin J Prev Med. 2015;49(7):26-30. 